\title{
Adaptive Special Strategies Resampling for Inertial-based Mobile Indoor Positioning Systems: An Initial Proposal
}

\author{
1,2, * Wan Mohd Yaakob Wan Bejuri, ${ }^{1}$ * Mohd Murtadha Mohamad, ${ }^{1}$ Raja Zahilah \\ Raja Mohd Radzi, ${ }^{1}$ Mazleena Salleh and ${ }^{1}$ Ahmad Fadhil Yusof. \\ ${ }^{1}$ Faculty of Computing, Universiti Teknologi Malaysia, MALAYSIA \\ ${ }^{2}$ Faculty of Information and Communication Technology, Universiti Teknikal \\ Malaysia, MALAYSIA \\ mr.wanmohdyaakob.my@ieee.org,murtadha@utm.my,zahilah@utm.my, \\ mazleena@utm,myandahmadfadhil@utm.my \\ *Corresponding author
}

\begin{abstract}
The phenomenon of sample impoverishment during particle filtering always contribute computation burden to the inertial-based mobile IPS systems. This is due to the factor of noise measurement and number of particle. Usually, the special strategies resampling algorithms was used. However, these algorithms just can fit in certain environment. This needs an adaptation of noise measurement and number of particle in a algorithm in order to make resampling with more intelligence, reliability and robust. In tbis paper, we will propose an adaptive special strategies resampling by adapting noise and particle measurement. These adaptation is used to determine the most suitable algorithm of special strategies resampling, that can be switched for resampling purpose. Finally, we illustrate our proposed solution our for indoor environment setup.
\end{abstract}

Keywords: Resampling , Sample Impoverishment, Indoor Positioning System

\section{Introduction}

With the rapid advancement in location technologies and tracking devices, and the demand for flawless solutions to overcome the problems associated with current mobile location based techniques, there is widespread interest in mobile IPS systems (IPS)[1-13]. One of the major components of mobile IPS is inertial-based positioning, which facilitates the tracing of individuals (or mobile nodes) within corridors or other enclosed structures by using inertial sensor. The inertial sensor can be used to determine user location everywhere and ubiquitous without using extra building infrastructure and it equipped in many smart phone nowadays [14-19]. Some examples of inertial-based mobile IPS include mobile asset navigation, mobile first-responder navigation and mobile emergency rescue and tracking [20-23]. One of the major issue of inertial-based mobile IPS, is sample impoverishment during particle filtering. This phenomenon will cause computation burden to the overall systems [24]. Two (2) reason behind this issue, whether it caused by the of small noise measurement in accurate sensor or small number of particle [25]. In order to counter the phenomenon, the special strategies resampling algorithm can be used. However, these special strategies resampling can survive in certain environment. Due to the inertial-based mobile IPS systems need to work in different kind of sensor and number of particle, it is benefit if it can survive with different environment. In tbis paper, we will be propose an adaptive special strategies resampling by adapting noise and particle measurement. These adaptation is to determine the most suitable algorithm of special strategies resampling that can be used as a resampling. The structure of the paper is as follows. Section 2 will present the basic concept related to inertial-based mobile IPS 
systems. Section 3 will present our problem formulation. Section 4 will present our objective of this paper. Section 5 will present our proposed method. Section 6 will present the special strategies resampling that will be used and manipulated in our proposed method. Section 7 will present our experiment setup. Finally, a discussion and the future direction of the paper will be provided in Section 8.

\section{Concept of Inertial-based Indoor Positioning on Mobile Sensing Platform}

Previous section discuss about the introduction of this paper. This section discusses the basic concept of inertial-based indoor positioning on mobile sensing platform. The concept of (see Figure 1 for fundamental system architecture) regards positioning determination across all environments [26] [27] [28] [29]. Usually, it requires a multisensor approach, augmenting standalone positioning with other signals, motion sensors, and environmental features [30] [31] [32].

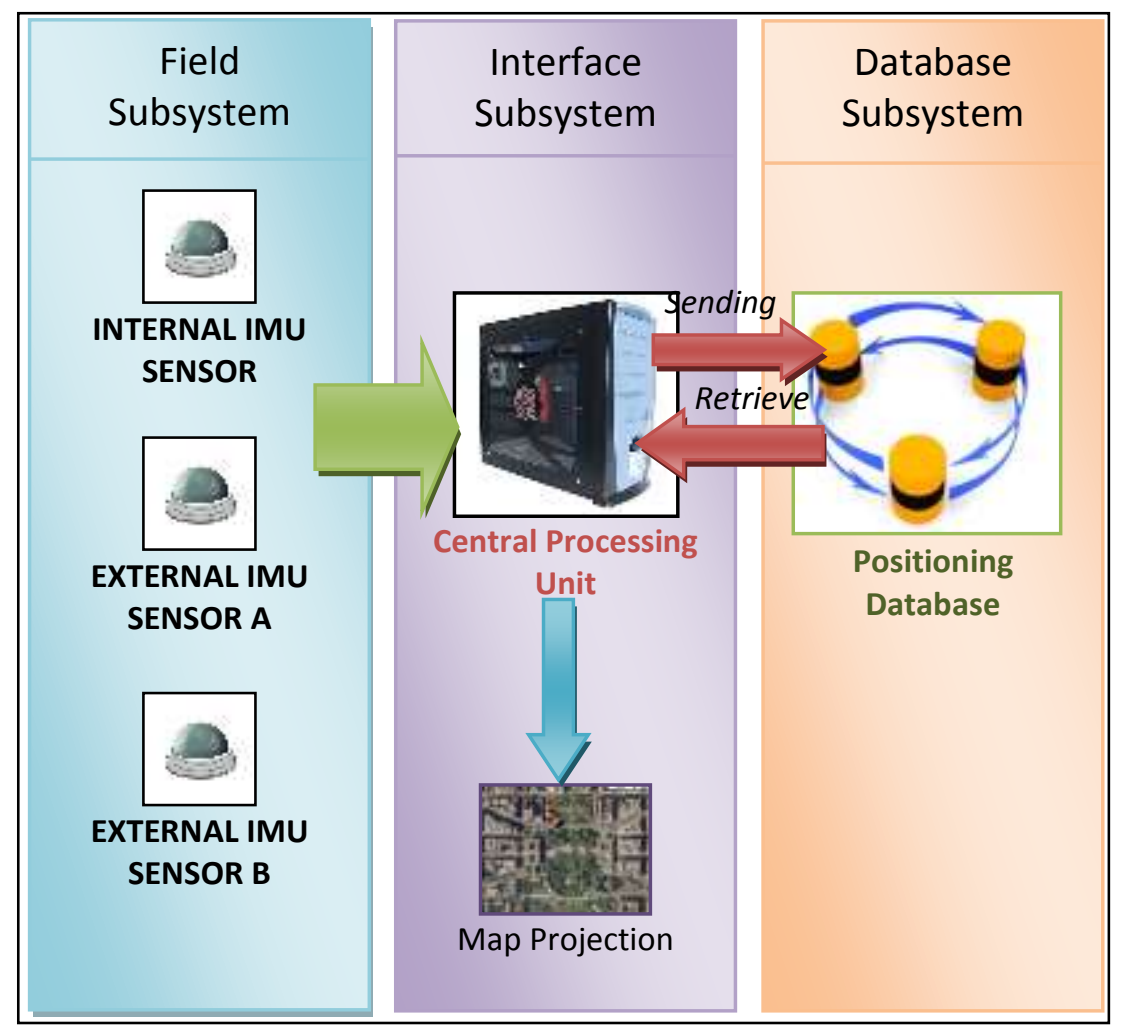

\section{Figure 1. Fundenmental System Architecture of Inertial-based Indoor Positioning on Mobile Sensing Platform}

This may be enhanced using three dimensional (3D) mapping, context awareness and cooperation between users. According to Figure 1, the inertial-based mobile IPS systems generally consist of three (3) subsystems (2), which are named as the field subsystem, the interface subsystem, and the database subsystem. In the normal situation, the inertial sensor will always continuously send their signal to the central processing unit (which located in interface subsystem). The received signals by then will be processed by a central processing unit (where the positioning algorithm is installed), before it is compared with the surveying data in the database server. Finally, the output of the system will display the mapping location on a mobile 
device screen. Next following section will discuss about problem formulation that we want to arise in this paper.

\section{Problem Formulation}

Previous section discuss about the basic concept of inertial-based indoor positioning on mobile sensing platform. This section discusses the. problem formulation that we arrised in this paper. The sample impoverishment phenomenon is a general issue in inertial-based mobile IPS systems (see Figure 2 for sample impoverishment phenomenon illustration). This phenomenon is a situation when very few particle have significant weight, while the rest was abandon during resampling process due to very small weight. This situation occurred during particle filtering and it is contribute to the computation burden to the overall system. According to pak 2016, the sample impoverishment is caused by small noise measurement and low particle number. The small noise measurement usually happened during software using accurate sensor, meanwhile low particle number usually happened in real time application.

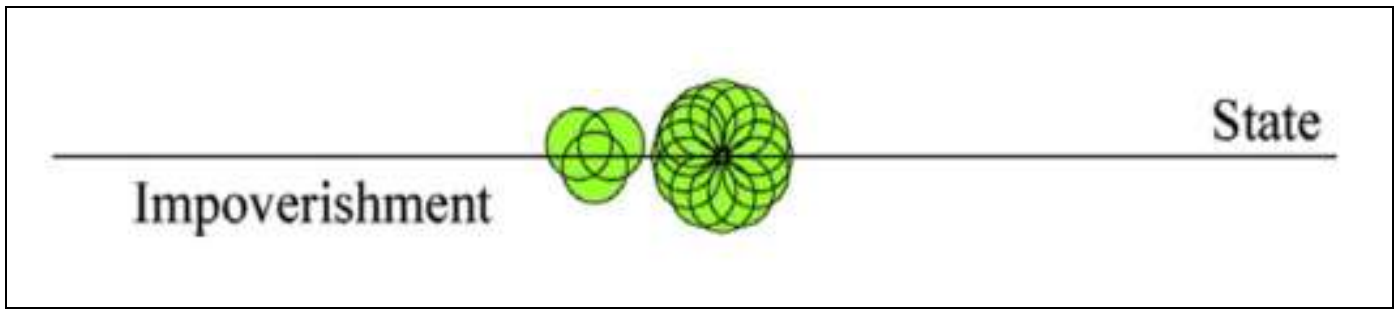

Figure 2. Illustration of Sample Impoverishment Phenomenon in Inertialbased Mobile IPS [33]

In order to counter this phenomenon, a special strategies resampling was used. Currently, there are three (3) common types, which are; modified resampling, variable resampling and roughening resampling. It can be say that modified resampling works by adjust the weight of particle (size of particle), variable resampling works by reduce the particle number, and meanwhile, the roughening resampling works by moving the particle that grouped in one direction. All of these is to split the particle distribution that grouped in one direction, so that it can be increase the diversity of particle distribution. As a general, these algorithms have their own purpose and capability (see the detail of algorithm capability in Table 1). It can be says the roughening resampling can be used to solve problem small noise measurement during particle filtering, the variable resampling can used to solve small particle number and the modified resampling can be used to solve problem normal noise measurement and big particle number. However, these algorithm just fit in certain environment (as mentioned before). If the environment changed, the algorithm cannot survive. Thus, it can be useful if the algorithm can be robust within these environment (normal and small noise measurement and small particle number). Next following section will discuss about the objective of this paper.

Table 1. Comparison of Special Strategies Resampling [] [] []

\begin{tabular}{|c|c|c|c|}
\hline Categorizations & $\begin{array}{c}\text { Modified } \\
\text { Resampling }\end{array}$ & $\begin{array}{c}\text { Variable } \\
\text { Resampling }\end{array}$ & $\begin{array}{c}\text { Roughening } \\
\text { Resampling }\end{array}$ \\
\hline Particle Size & $\begin{array}{c}\text { Big } \\
{[34][35][36]}\end{array}$ & Small [37] & Big [37] \\
\hline $\begin{array}{c}\text { Noise } \\
\text { Measurement }\end{array}$ & Normal [34] [10] & Normal [25] & Small or Normal [25] \\
\hline
\end{tabular}




\section{Objective}

Previous section discuss about the problem formulation that we arrised in this paper. This section discusses the objective of this paper. As objective of this paper, the study aims to outline a credible new special strategies resampling based on the adaptation of noise measurement and particle number. By implementing this algorithm, it should be possible to ascertain counter the sample impoverishment in different environment. Additionally, it might be possible to robust in different implementation of inertial-based mobile IPS systems [38] [39]. The results of the study could also significantly contribute to modernising current location detection systems, and provide useful finding for other inertial-based positioning systems studies [40] [41] [42] [43]. Next following section will discuss about the proposed method of special strategies resampling algorithm.

\section{Adaptive Noise and Particle based Special Strategies Resampling}

Previous section discuss about the objective this paper. As mentioned before, the aim of this paper is to propose a new special strategies resampling,based on the adaptation of noise measurement and particle number. In this section, we will discusses about the proposed method of special strategies resampling algorithm. In our proposed method, there are three (3) type of situation that will be focused, which are; the situation during inertial-based mobile IPS system using internal inertial sensor, using external inertial sensor and real time application. The reason behind is, these situations is causing sample impoverishment for positioning determination. According to Figure 2, it shows the block diagram of adaptive noise and particle based special strategies resampling. Initially, the system will receive the inertial data signal from sensor. At first, the noise measurement will be measured. If the noise measurement is high, the algorithm will do resampling based on roughening resampling. But, if the noise measurement is low, the particle number will be measured. Finally, the algorithm will be do resampling based on modified resampling (if the particle is high) or variable resampling (if the particle is low). Next following section will discuss about the special strategies resampling algorithm that will be used and manipulated in our proposed method. 


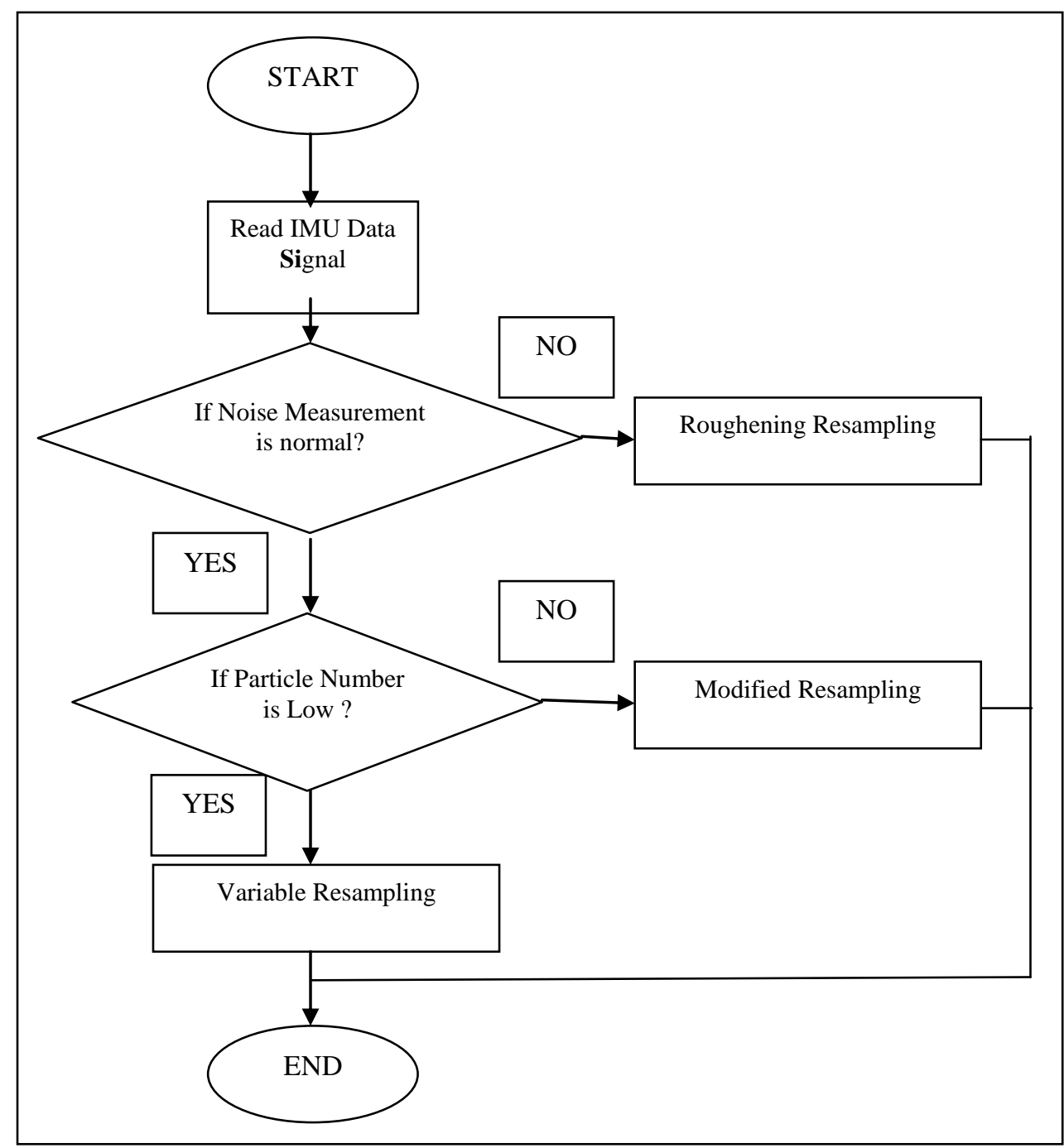

Figure 3. Block Diagram of Adaptive Noise and Particle based Special Strategies Resampling

\section{Roughening, Modified and Variable Resampling}

Previous section discuss about the proposed method of special strategies resampling algorithm. This section discuss about the special strategies resampling algorithm that will used and manipulated in our proposed method. In our proposed method, it can be say that these special strategies resampling will be choosen based on noise measurement and particle number criteria. Three (3) types of special strategies resampling will be used as a choice for reducing particle impoverishment phenomenon which are; roughening resampling, modified resampling and variable resampling [44] [45] [33] [46] . In this section, we will describe about how our algorithm work when it need to resample based on roughening resampling, modified resampling and variable resampling. At first we will discuss about resampling based on roughening. Roughening will move the particle location by spread the distribution. After initialization at time $t=1$, our algorithm proceeds with a rejuvenation at each subsequent integer time. The rejuvenation comprises two steps: a resample step and a move step. As initial, the rejuvenation will do initialization at time $t=1$, and generate the initial set of particles $S_{1}$ by sampling, independently for $j=1, \ldots . n_{1}$, 


$$
\theta_{1}^{(j)} \sim \pi_{1}
$$

where $\sim$ denotes 'is sampled from'. Then, for $k=1,2, \ldots$, we have the rejuvenation step. After initialization, the process of rejuvenation will take place. At each time $=k+1$, it will calculate weights $w_{k}^{(i)}$, for $i=1, \ldots, n_{k}$ and then generate $S_{k+1}$ by performing the following two (2) steps, independently for $j=1 \ldots \ldots, n_{k+1}$. The step is known as resample and move. The step is following by random selection of particle from $S_{k}$, (such that $\theta_{k}^{(i)}$ is selected with probability proportional to $w_{k}^{(i)}$, for $i=, \ldots, n_{k}$, and denote the selected particle by $\theta_{k}^{(i j)}$ ), and move $\theta_{k}^{(i j)}$ to a new position $\theta_{k+1}^{(i)}$ by sampling.

Secondly, we describe about resample based on modified resampling. In modified resampling, all the particles are resampled based on their probability, $p_{t}^{[m]}$. The probabilities are generally equivalent to the weight of the particles, $w_{t}^{[m]}$. However, generally, the particles having probabilities as the function of their weights are drawn, for instance;

$$
p_{t}^{(m)} \propto\left(w_{t}^{(m)}\right)^{\alpha}
$$

Where in $\alpha>0$. If, $0<\alpha<1$, it is seen that the low-weight particles are boosted further and the particles with large weights display suppressed probabilities, thus, improving the diversity of the particles. Contrastingly, $\alpha>1$ ensures that there is a higher preference for the high-weight particles. Also, using the auxillary weights, one can implement the knowledge with regards to the subsequent observation before carrying out the particle resampling. Thus, particles having better probabilities are more likely to survive. The step involving generation of the auxillary variables that represents the particle fitness is seen as the resampling step which considers the instant future and the present state while selecting particles. The idea of fusing the data obtained from the recent observations along with the present weights while selecting the particles is very commendable.

Finally, we describe about resample based on variable resampling. In variable resampling, It is helps in determining the required particle number depending on the KLD observed between the distribution of interests and the sample based maximum likelihood estimates. The needed, $N$, particle number, is determined to have a probability value of $1-\rho$. It is seen that the KLD present between the distribution of interests and the sample based maximum likelihood estimates for the desired particle distribution is lower than the pre-specified error-bound threshold value of $\mathrm{f}$. It is seen that;

$$
\begin{aligned}
& \text { Where } N=\frac{1}{2 \varepsilon} q \\
& q=F^{-1}(1-\rho)
\end{aligned}
$$

Wherein, $F^{-1}($.$) represents the inverse of cumulative chi-squared distribution having a$ $k-1$ degree of freedom, while $\mathrm{k}$ refers to the bin number [non-overlapping (multi)dimensional interval] that is used for particle sorting. Nvalue in Equation (3) can be computed approximately. However, practically, the particle number in resampling could be hard-pressed to exceed the minimal threshold value. Generally, the posterior of the state is ideally used as the desired distribution. This posterior can be calculated by using the method of predictive distribution. In theory, it is more difficult and more flexible to use Equation (3) while resampling instead of sampling, which then leads to the KLD-resampling technique. In the KLD technique, the particle resampling is conducted individually till the needed amount defined by Equation (3) is achieved. Next following section will discuss about the experiment setup of the proposed method. 


\section{Experiment Setup}

The previous section discussed the resampling selection when it chosen. This section discusses the experiment setup of the our proposed adaptive noise measurement and particle special strategies resampling. Currently, a number of simulations will be conducted to study the performance of the proposed algorithm. The experiment will be simulated with the parameters are set as follows. The process and measurement noise covariance matrices are taken as;

$$
\begin{gathered}
\sigma_{w}=0.1 \\
\sigma_{v}=0.5
\end{gathered}
$$

To allow a fair comparison, a comprehensive quantitative comparison of the resampling methods should be considered through the use of three (3) situation in bearing tracking problem (see Table 2 parameter setting for experiment setup).

Table. 2. Parameter Setting for Experiment Setup [25]

\begin{tabular}{|c|c|c|c|c|}
\hline Situation & $\begin{array}{c}\text { Measureme } \\
\text { nt Noise }\end{array}$ & $\begin{array}{c}\text { Proces } \\
\text { s Noise }\end{array}$ & $\begin{array}{c}\text { Parti } \\
\text { cle } \\
\text { Number }\end{array}$ & $\begin{array}{c}\text { Performance } \\
\text { Metric }\end{array}$ \\
\hline $\begin{array}{l}\text { Situation 1: } \\
\text { Positioning using } \\
\text { low cost inertial } \\
\text { sensor devices }\end{array}$ & 0.1 & 0.5 & 500 & $\begin{array}{c}\bullet \quad \text { Estimated } \\
\text { positioning. } \\
\bullet \quad \text { Localization } \\
\text { error }\end{array}$ \\
\hline $\begin{array}{l}\text { Situation 2: } \\
\text { Positioning using } \\
\text { accurate inertial } \\
\text { sensor devices }\end{array}$ & 0.1 and 0.05 & 0.5 & 500 & $\begin{array}{l}\bullet \text { Estimated } \\
\text { positioning. } \\
\text { Localization error }\end{array}$ \\
\hline $\begin{array}{l}\text { Situation 3: Fast } \\
\text { positioning using } \\
\text { small particle } \\
\text { quantity }\end{array}$ & 0.1 & 0.5 & $\begin{array}{c}50 \\
\text { and } 10\end{array}$ & $\begin{array}{l}\text { Estimated } \\
\text { positioning } \\
\bullet \\
\text { error Localization }\end{array}$ \\
\hline
\end{tabular}

The situation is; positioning using low cost inertial sensor devices, positioning using accurate inertial sensor devices, and fast positioning using small particle quantity. This is to establish whether the proposed algorithm can survive in a different environment of sample impoverishment. For the first $\left(1^{\text {st }}\right)$ situation, the algorithm will be simulated using generated particle is 500 and the initial position is unknown. For the second $\left(2^{\text {nd }}\right)$ situation (positioning using accurate inertial sensor devices), it will be simulated in two (2) different generated noise measurement which are $\sigma_{v}=0.1$ and $\sigma_{v}=0.05$. For the third $\left(3^{\text {rd }}\right)$ situation, it will be simulated in small particle number, which are; $N=50$ and $N=10$. Next following section will discuss about the conclusion and future works.

\section{Conclusions and Future Directions}

The previous section discussed the experiment setup of our proposed method. This current section, discusses the conclusions. This paper discuss about how to solve sample impoverishment phenomenon during particle filtering in inertial-based mobile IPS system in different environment of noise measurement and number of particle. This needs an adaptation of noise measurement and number of particle in algorithm for resampling with more intelligence, reliability and robust. Thus, an adaptive special strategies resampling by adapting noise and particle measurement has been proposed. These adaptation is to determine the most suitable algorithm of special strategies resampling that can be used, in order to counter this phenomenon. Finally, our proposed solution our for indoor 
environment setup has been illustrated. As future work, we will continue our experiment by using this result and simulate our proposed method in order to obtain more valuable findings.

\section{Acknowledgements}

These should be brief and placed at the end of the text before the references. This paper was inspired from my $\mathrm{Ph}$.D proposal which is related to beacon selection method in FMradio positioning system. The author would also like to thank our supervisor Dr. Mohd Murtadha Mohamad for their insightful comments on earlier drafts of this paper.

\section{Refereces}

[1] W. M. Y. W. Bejuri, M. M. Mohamad and R. Z. R. M. Radzi, "Optimisation of Emergency Rescue Location (ERL) using KLD-Resampling: An Initial Proposal”, Int. J. U- E-Serv. Sci. Technol., vol. 9, no. 2, (2016), pp. 249-262.

[2] F. C. Teixeira, J. Quintas, P. Maurya and A. Pascoal, "Robust particle filter formulations with application to terrain-aided navigation", Int. J. Adapt. Control Signal Process., (2016).

[3] W. M. Y. W. Bejuri, M. M. Mohamad, R. Z. R. M. Radzi, M. Salleh and A. F. Yusof, "Adaptive Memory Size Based Fuzzy Control for Mobile Pedestrian Navigation", in Lecture Notes on Data Engineering and Communications Technologies, vol. 5, Springer, Cham, (2017), pp. 132-140.

[4] W. M. Y. W. Bejuri, M. M. Mohamad, R. Z. R. M. Radzi, M. Salleh and A. F. Yusof, "Adaptive Resampling for Emergency Rescue Location: An Initial Concept", in Lecture Notes in Electrical Engineering, vol. 425, Springer, Singapore, (2017), pp. 269-273.

[5] W. M. Y. W. Bejuri, M. M. Mohamad and R. Zahilah, "A Proposal of Emergency Rescue Location (ERL) using Optimization of Inertial Measurement Unit (IMU) based Pedestrian Simultaneously Localization and Mapping (SLAM) ”, Int. J. Smart Home, vol. 9, no. 12, (2015), pp. 9-22.

[6] W. M. Y. W. Bejuri, M. M. Mohamad, R. Z. R. M. Radzi, M. Salleh and A. F. Yusof, "A Proposal of Location Aware Shopping Assistance Using Memory-Based Resampling”, in Lecture Notes in Electrical Engineering, vol. 425, Springer, Singapore, (2017), pp. 482-486.

[7] W. M. Y. W. Bejuri, M. M. Mohamad and R. Zahilah, "Emergency Rescue Localization (ERL) using GPS, Wireless LAN and Camera”, Int. J. Softw. Eng. Its Appl., vol. 9, no. 9, (2015), pp. 217-232.

[8] W. M. Y. W. Bejuri, M. M. Mohamad, M. Sapri and M. A. Rosly, "Investigation of Color Constancy for Ubiquitous Wireless LAN/Camera Positioning: An Initial Outcome”, Int. J. Adv. Comput. Technol. IJACT, vol. 4, no. 7, (2012), pp. 269-280.

[9] W. M. Y. W. Bejuri, M. M. Mohamad and R. Zahilah, "Offline Beacon Selection-Based RSSI Fingerprinting for Location-Aware Shopping Assistance: A Preliminary Result", in New Trends in Intelligent Information and Database Systems, Springer International Publishing, (2015), pp. 303-312.

[10] W. M. Y. W. Bejuri, M. M. Mohamad, Zahilah and Raja, "Optimisation of Emergency Rescue Location (ERL) using KLD Resampling: An Initial Proposal”, Int. J. U- E- Serv. Sci. Technol., vol. 9, no. 2, (2016), pp. 249-262.

[11] W. M. Y. W. Bejuri, M. M. Mohamad and R. Zahilah, "Optimization of Rao-Blackwellized Particle Filter in Activity Pedestrian Simultaneously Localization and Mapping (SLAM): An Initial Proposal”, Int. J. Secur. Its Appl., vol. 9, no. 11, (2015), pp. 377-390.

[12] W. M. Y. W. Bejuri and M. M. Mohamad, "Performance analysis of grey-world-based feature detection and matching for mobile positioning systems", Sens. Imaging, vol. 15, no. 1, (2014), p. 95.

[13] W. M. Y. W. Bejuri, M. M. Mohamad, M. Sapri and M. A. Rosly, "Performance Evaluation of Mobile U-Navigation based on GPS/WLAN Hybridization", J. Converg. Inf. Technol. JCIT, vol. 7, no. 12, (2012), pp. 235-246.

[14] W. M. Y. W. Bejuri, M. M. Mohamad, M. Sapri, M. S. M. Rahim and J. A. Chaudry, "Performance Evaluation of Spatial Correlation-based Feature Detection and Matching for Automated Wheelchair Navigation System”, Int. J. Intell. Transp. Syst. Res., vol. 12, no. 1, (2014), pp. 9-19.

[15] Y. Li, Y. Zhuang, P. Zhang, H. Lan, X. Niu and N. El-Sheimy, "An improved inertial/wifi/magnetic fusion structure for indoor navigation”, Inf. Fusion, vol. 34, (2017), pp. 101-119.

[16] W. M. Y. W. Bejuri, M. M. Mohamad and M. Sapri, "Ubiquitous Positioning: A Taxonomy for Location Determination on Mobile Navigation System”, Signal Image Process. Int. J. SIPIJ, vol. 2, no. 1, (2011), pp. 24-34.

[17] W. M. Y. W. Bejuri, W. M. N. W. M. Saidin, M. M. Mohamad, M. Sapri and K. S. Lim, "Ubiquitous Positioning: Integrated GPS/Wireless LAN Positioning for Wheelchair Navigation System" in Intelligent Information and Database Systems, vol. 7802, (2013), pp. 394-403.

[18] W. M. Y. W. Bejuri, M. M. Mohamad, M. Sapri and M. A. Rosly, "Ubiquitous WLAN/Camera Positioning using Inverse Intensity Chromaticity Space-based Feature Detection and Matching: A 
Preliminary Result”, in International Conference on Man-Machine Systems 2012 (ICOMMS 2012), (2012).

[19] W. M. Y. W. Bejuri and M. M. Mohamad, "Wireless LAN/FM Radio-based Robust Mobile Indoor Positioning: An Initial Outcome”, Int. J. Softw. Eng. Its Appl., vol. 8, no. 2, (2014), pp. 313-324.

[20] W. M. Y. W. Bejuri, M. M. Mohamad and R. Zahilah, "Optimization of Rao-Blackwellized Particle Filter in Activity Pedestrian Simultaneously Localization and Mapping (SLAM): An Initial Proposal”, Int. J. Secur. Its Appl., vol. 9, no. 11, (2015), pp. 377-390.

[21] W. M. Y. W. Bejuri, M. M. Mohamad, R. Zahilah and R. M. Radzi, "Emergency Rescue Localization (ERL) using GPS, Wireless LAN and Camera", Int. J. Softw. Eng. Its Appl., vol. 9, no. 9, (2015), pp. 217-232.

[22] W. M. Y. W. Bejuri, M. M. Mohamad and R. Z. R. M. Radzi, "Offline Beacon Selection-Based RSSI Fingerprinting for Location-Aware Shopping Assistance: A Preliminary Result", in New Trends in Intelligent Information and Database Systems, vol. 598, Springer, (2015), pp. 303-312.

[23] W. M. Y. W. Bejuri and M. M. Mohamad, "Performance Analysis of Grey-World-based Feature Detection and Matching for Mobile Positioning Systems”, Sens. Imaging, vol. 15, no. 1, (2014), pp. 124.

[24] P. Vadakkepat and L. Jing, "Improved particle filter in sensor fusion for tracking randomly moving object”, IEEE Trans. Instrum. Meas., vol. 55, no. 5, (2006), pp. 1823-1832.

[25] J. M. Pak, C. K. Ahn, Y. S. Shmaliy, P. Shi and M. T. Lim, "Accurate and Reliable Human Localization Using Composite Particle/FIR Filtering”, IEEE Trans. Hum.-Mach. Syst., (2016).

[26] S.-H. Jung, G. Lee and D. Han, "Methods and Tools to Construct a Global Indoor Positioning System", IEEE Trans. Syst. Man Cybern. Syst., (2017).

[27] X. Li, D. Wei, Q. Lai, Y. Xu and H. Yuan, "Smartphone-based integrated PDR/GPS/Bluetooth pedestrian location”, Adv. Space Res., vol. 59, no. 3, (2017), pp. 877-887.

[28] Y. Li, Y. Zhuang, P. Zhang, H. Lan, X. Niu and N. El-Sheimy, "An improved inertial/wifi/magnetic fusion structure for indoor navigation”, Inf. Fusion, vol. 34, (2017), pp. 101-119.

[29] Q. Liu, J. Qiu and Y. Chen, "Research and development of indoor positioning", China Commun., vol. 13, no. Supplement2, pp. 67-79.

[30] H. Ren, P. Chai, Y. Zhang, D. Xu, T. Xu and X. Li, "Semiautomatic indoor positioning and navigation with mobile devices", Ann. GIS, (2017), pp. 1-12.

[31] M. V. Albert, I. Shparii and X. Zhao, "The Applicability of Inertial Motion Sensors for Locomotion and Posture", in Locomotion and Posture in Older Adults, Springer, (2017), pp. 417-426.

[32] G. Retscher and F. Roth, "Wi-Fi Fingerprinting with Reduced Signal Strength Observations from LongTime Measurements", in Progress in Location-Based Services 2016, Springer, (2017), pp. 3-25.

[33] T. Li, T. P. Sattar and S. Sun, "Deterministic resampling: unbiased sampling to avoid sample impoverishment in particle filters", Signal Process., vol. 92, no. 7, (2012), pp. 1637-1645.

[34] N. Kwak, I.-K. Kim, H.-C. Lee and B.-H. Lee, "Adaptive prior boosting technique for the efficient sample size in FastSLAM", in Intelligent Robots and Systems, 2007. IROS 2007. IEEE/RSJ International Conference on, (2007), pp. 630-635.

[35] I. Kim, N. Kwak, H. Lee and B. Lee, "Improved particle filter using geometric relation between particles in FastSLAM-ERRATUM”, Robotica, vol. 27, no. 6, (2009), pp. 959-959.

[36] T. M. Sanguino and F. P. Gómez, "Evaluation of a dispersion-based adaptive strategy using KinectTM and dynamic particle filter", in Information Fusion (FUSION), 2014 17th International Conference on, (2014), pp. 1-8.

[37] T. de J. M. Sanguino and F. P. Gómez, "Toward Simple Strategy for Optimal Tracking and Localization of Robots With Adaptive Particle Filtering”, IEEEASME Trans. Mechatron., vol. 21, no. 6, (2016), pp. 2793-2804.

[38] N. J. Gordon, D. J. Salmond and A. F. Smith, "Novel approach to nonlinear/non-Gaussian Bayesian state estimation", in IEE Proceedings F-Radar and Signal Processing, vol. 140, (1993), pp. 107-113.

[39] G. Falco, M. Pini and G. Marucco, "Loose and Tight GNSS/INS Integrations: Comparison of Performance Assessed in Real Urban Scenarios", Sensors, vol. 17, no. 2, (2017), p. 255.

[40] Z. Li, J. Gao, J. Wang and Y. Yao, "PPP/INS tightly coupled navigation using adaptive federated filter", GPS Solut., vol. 21, no. 1, (2017), pp. 137-148.

[41] I. Belhajem, Y. B. Maissa and A. Tamtaoui, "An Improved Robust Low Cost Approach for Real Time Vehicle Positioning in a Smart City", in Industrial Networks and Intelligent Systems, Springer, (2017), pp. 77-89.

[42] A. Ghetti, L. Vittuari and M. Zanzi, "A Satellite and Inertial Navigation Solution in Crises Management Operation for First Responder Application", in Advances in Science and Technology, vol. 101, (2017), pp. $158-167$.

[43] H. Xing, J. Li, B. Hou, Y. Zhang and M. Guo, "Pedestrian Stride Length Estimation from IMU Measurements and ANN Based Algorithm", J. Sens., vol. 2017, (2017).

[44] T. Li, G. Villarrubia, S. Sun, J. M. Corchado and J. Bajo, "Resampling methods for particle filtering: identical distribution, a new method, and comparable study", Front. Inf. Technol. Electron. Eng., vol. 16, no. 11, (2015), pp. 969-984. 
International Journal of Control and Automation

Vol. 10, No. 10 (2017)

[45] T. Li, S. Sun and T. P. Sattar, "Adapting sample size in particle filters through KLD-resampling", Electron. Lett., vol. 49, no. 12, (2013), pp. 740-742.

[46] T. Li, M. Bolic and P. M. Djuric, "Resampling methods for particle filtering: classification, implementation, and strategies", IEEE Signal Process. Mag., vol. 32, no. 3, (2015), pp. 70-86. 